\title{
Case Report: Four Cases of Cortical/Brainstem Encephalitis Positive for Myelin Oligodendrocyte Glycoprotein Immunoglobulin G
}

OPEN ACCESS

Edited by:

Jodie Burton,

University of Calgary, Canada

Reviewed by:

Frederic London,

Catholic University of

Louvain, Belgium

Jefferson Becker,

Pontifical Catholic University of Rio

Grande do Sul, Brazil

*Correspondence:

Shaohua Yin

yinsh1012@163.com

Specialty section:

This article was submitted to

Multiple Sclerosis and

Neuroimmunology,

a section of the journal

Frontiers in Neurology

Received: 13 September 2021 Accepted: 20 December 2021

Published: 21 January 2022

Citation:

Wang W, Yin J, Fan Z, Kang J, Wei J,

Yin $X$ and Yin S (2022) Case Report:

Four Cases of Cortical/Brainstem

Encephalitis Positive for Myelin

Oligodendrocyte Glycoprotein Immunoglobulin $\mathrm{G}$.

Front. Neurol. 12:775181

doi: 10.3389/fneur.2021.775181

\author{
Wan Wang ${ }^{1}$, Juntao Yin ${ }^{2}$, Zhiliang Fan ${ }^{1}$, Juxian Kang ${ }^{1}$, Jia Wei ${ }^{1}$, Xiaoqian Yin ${ }^{3}$ and \\ Shaohua Yin ${ }^{1 *}$ \\ ${ }^{1}$ Department of Neurology, Affiliated Hospital Xingtai People's Hospital, Hebei Medical University, Xingtai, China, \\ ${ }^{2}$ Department of Neurology, Xingtai Third Hospital, Xingtai, China, ${ }^{3}$ Department of Imaging, Affiliated Hospital Xingtai People's \\ Hospital, Hebei Medical University, Xingtai, China
}

Aim: Despite a significant improvement in the number of studies on myelin oligodendrocyte glycoprotein (MOG)-immunoglobulin G ( $(\mathrm{gG})$-associated disorder (MOGAD) over the past few years, MOG-IgG-associated cortical/brainstem encephalitis remains a relatively uncommon and less-reported presentation among the MOGAD spectrum. This study aimed to report the clinical course, imaging features, and therapeutic response of MOG-lgG-associated cortical/brainstem encephalitis.

Methods: Data of four patients who suffered from cortical encephalitis with epileptic seizures and/or brainstem encephalitis during the course of the disease were retrospectively collected and analyzed.

Results: In this study, three male patients and one female patient, with a median age of onset of 21 years (ranging 20-51 years) were enrolled. An epileptic seizure was the main symptom of cortical encephalitis in these patients, while the manifestations of brainstem encephalitis were diverse. Cranial MRI demonstrated abnormal signals in unilateral or bilateral cortical or brainstem. Cerebrospinal fluid studies showed normal or mildly elevated leukocyte counts and protein levels, and a cell-based assay detected positive MOG-lgG in the serum of all patients. Two patients were misdiagnosed at the first attack, and both experienced a relapse. All of them accepted the first-line immunotherapy after a confirmed diagnosis and had a good outcome.

Conclusion: Early suspicion of MOG-IgG-associated encephalitis is necessary for any patient with sudden onset of seizures or symptoms of brainstem damage, especially with lesions on unilateral/bilateral cortical or brainstem on brain MRI.

Keywords: brainstem encephalitis, cortical encephalitis, epileptic seizures, myelin oligodendrocyte glycoprotein, neuromyelitis optica spectrum disorder

\section{INTRODUCTION}

Myelin oligodendrocyte glycoprotein (MOG) is a myelin protein expressed on the outer surface of myelin sheaths and oligodendrocyte processes in the central nervous system (CNS) (1). Although MOG-immunoglobulin G (IgG)-positive cases account for about $25 \%$ of aquaporin4 (AQP4)-seronegative neuromyelitis optica spectrum disorders (2), the clinical manifestation 
is less well-defined. In recent years, an enormous amount of research has been conducted to determine the role of MOGIgG in a wide clinical spectrum of inflammatory demyelinating CNS disorders, including optic neuritis $(\mathrm{ON})$, myelitis, cortical damage (3), and less commonly, brainstem lesions (4). Compared with $\mathrm{ON}$ and myelitis, cortical encephalitis with seizures and brainstem encephalitis are emerging presentations of MOG-IgGassociated disorder (MOGAD), and the related research has been rarely reported. The relationship between MOG-IgG and cortical encephalitis presentation was not recognized until the first case reported in 2017 (5). Subsequent case series further confirmed the association between MOG-IgG, fluid-attenuated inversion recovery (FLAIR)-hyperintense cerebral cortical lesions, and sudden epilepsy. Furthermore, most patients with MOG-IgGassociated cortical encephalitis had a relapsing disease course and experienced other demyelinating events, such as ON, myelitis, or brainstem damage, at a certain stage of the disease (6-10).

In the present study, four cases of MOG-IgG-associated cortical/brainstem encephalitis are reported, providing detailed information on the clinical manifestations, imaging features, disease evolution, and treatment outcomes of this disease.

\section{CASE PRESENTATION}

\section{Case 1}

A previously healthy 20 -year-old male patient presented with a witnessed primary generalized tonic-clonic seizure. After about $10 \mathrm{~h}$ in a coma, a nervous system examination revealed no limb weakness or sensory symptoms. Poorly marginated, hyperintense lesions in the left frontotemporal parietal lobe and the right frontal lobe were displayed on both FLAIR (Figures 1A, 2A-E) and T2-weighted images of cranial MRI (Figure 1C), which were less evident on T1-weighted (Figure 1B) and diffusionweighted images (Figure 1D). No epileptic waves were captured on an electroencephalogram (EEG). Laboratory data revealed an increase in the white blood cell count $\left(17.32 \times 10^{9} / \mathrm{L}\right)$. A cerebrospinal fluid (CSF) analysis showed a mild increase in leukocytes $(80 / \mu \mathrm{l})$ and protein $(48 \mathrm{mg} / \mathrm{dl})$. CSF cytology suggested lymphocytic reaction. CSF culture was negative. Neither immunoglobulin M (IgM) nor IgG of the herpes simplex virus were tested positive in CSF. The patient was diagnosed with viral encephalitis and treated with dexamethasone and acyclovir combined with antiepileptic drugs (sodium valproate) for 2 weeks. He no longer had epileptic seizures and was advised to continue prednisone [ $40 \mathrm{mg}$ qd (once a day)] orally for 40 days after discharge. The dosage of prednisone was reduced by $5 \mathrm{mg}$ every 5 days until discontinued. However, he had to be readmitted several times within 4 months because of frequent epileptic seizures and headaches with or without a fever. During this period, seizures occurred in two main forms: primary generalized and focal. He received symptomatic treatment each time. A follow-up brain MRI after 3 months suggested that FLAIR hyperintensities in the bilateral cortical regions were shallow and fewer (Figures 1E, 2F). He was readmitted because of dizziness and unsteady gait 3 years later, and a nervous system examination revealed poor left-side ataxia. Cranial MRI revealed FLAIR-hyperintense (Figure 1F), T1-hyperintense (Figure 1G), T2-hyperintense (Figure 1H), and diffusion-hyperintense (Figure 1I) lesions in the pons and left brachium pontis. CSF analysis showed four leukocytes and a mildly increased protein level $(51 \mathrm{mg} / \mathrm{dl})$. AQP4-IgG and oligoclonal bands in serum and CSF were negative. The myelin basic protein (MBP) level in the serum and CSF was not elevated. Antibodies of autoimmune encephalitis in the serum and CSF were negative. The cell-based assays (CBAs) revealed that MOGIgG was positive with titers of 1:10 in the serum. Thus, the diagnosis was corrected to MOG-IgG-associated encephalitis. He received intravenous pulse methylprednisolone $(500 \mathrm{mg} /$ day for 3 days), followed by oral prednisone ( $60 \mathrm{mg} \mathrm{qd}$, with a tapering dose of $5 \mathrm{mg}$ every 1 week) and mycophenolate mofetil [0.75 $\mathrm{g}$ bid (twice a day)]. The FLAIR hyperintensities in the brainstem were shallow and smaller after 1 month (Figure 1J). The dosage of prednisone was gradually reduced to $10 \mathrm{mg}$ daily and on maintenance for 1 year. He fully recovered, and MOG-IgG in his serum turned negative after 18 months. No recurrent events occurred during a 28-month follow-up.

\section{Case 2}

A 20-year-old male patient without any previous medical history was admitted to the hospital with twice primary generalized tonic-clonic seizures in $48 \mathrm{~h}$. The neurological examination was normal. The brain MRI showed FLAIR-hyperintense lesions in the left frontal lobe (Figures 2G-K). EEG captured 2- to $3-\mathrm{Hz}$ complex waves of sharp slow frequencies in the right frontal and bilateral occipitotemporal lobes. The laboratory data revealed a normal white blood cell count and blood biochemistry results. The CSF study showed a mild increase in leukocytes $(31 / \mu \mathrm{l})$ and a normal protein level. The antibodies of autoimmune encephalitis and CNS demyelinating disease in the serum and CSF were tested, which indicated non-elevated MBP levels and negative oligoclonal bands and AQP4-IgG. However, a CBA revealed antiMOG-IgG in his serum (1:100). He was diagnosed with MOGIgG-associated encephalitis and treated with antiepileptic drugs (levetiracetam) and intravenous pulse methylprednisolone (500 $\mathrm{mg} /$ day for 3 days), followed by oral prednisone (60 mg qd) and mycophenolate mofetil ( $0.5 \mathrm{~g}$ bid) induction. The FLAIR hyperintensities in the unilateral cortical regions disappeared after 1 month (Figure 2L). With a tapering dose of $5 \mathrm{mg}$ prednisone every 1 week and low-dose prednisone (10 mg daily) for 6 months, the patient did not experience any relapse during the 16-month follow-up.

\section{Case 3}

A 51-year-old female patient was admitted to the hospital for left-side facial paralysis and left perioral numbness. Her medical history showed hysterectomy because of uterine fibroids for 15 years. A neurological examination on admission showed left-side peripheral facial paralysis and decreased touch and pain sensation in the left perioral area. Cranial MRI disclosed nodular FLAIR-hyperintense (Figure 3A), T1-hypointense (Figure 3B), T2-hyperintense (Figure 3C), and diffusionhyperintense (Figure 3D) lesions in the left brachium pontis, with an enhanced edge on T1-weighted images (Figure 3E). No supratentorial lesion was found. The conventional laboratory tests of blood and serum antineutrophil cytoplasmic antibody and anti-myeloperoxidase antibody were negative. The CSF 

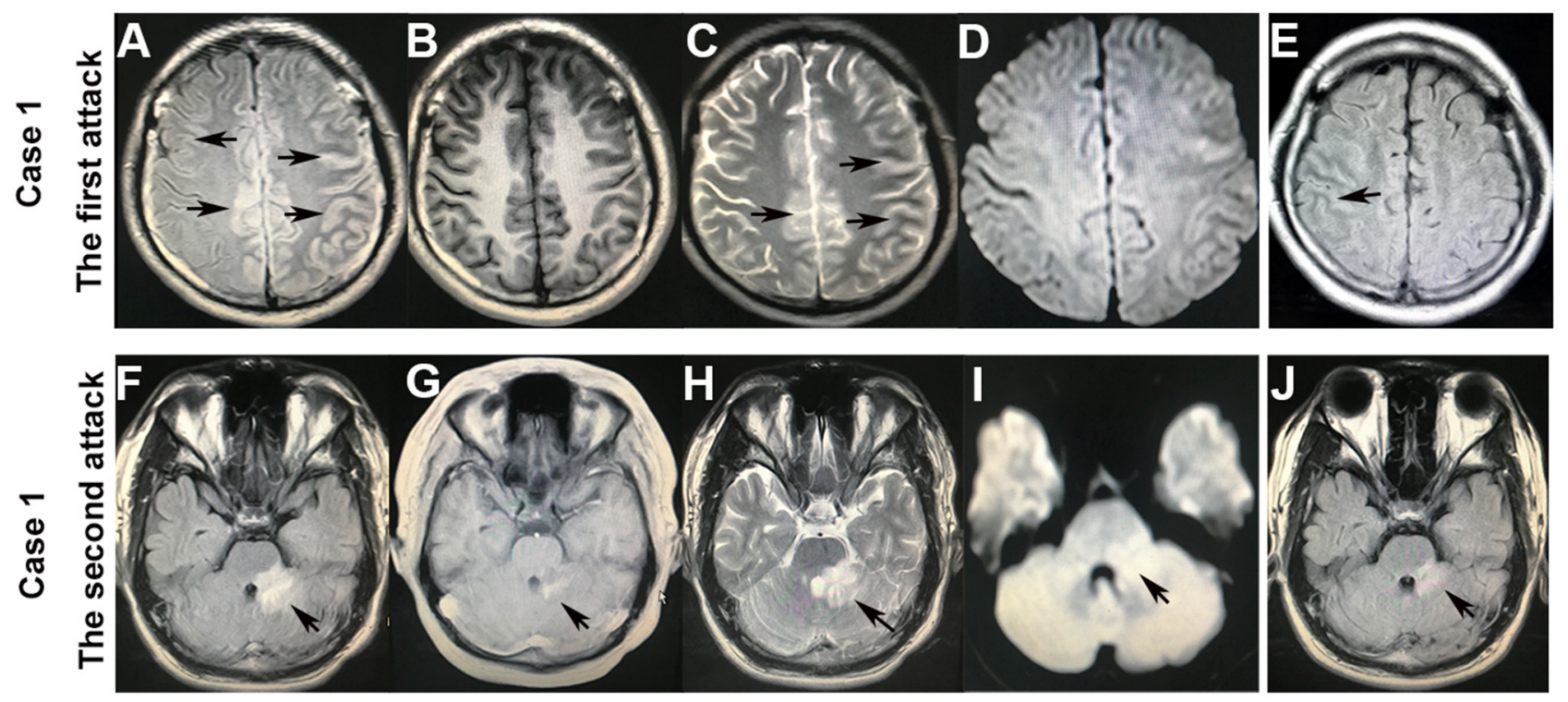

FIGURE 1 | Brain MRI of case 1. After the first attack of an epileptic seizure, the hyperintense lesions with a hazy border in the bilateral cortex on fluid-attenuated inversion recovery (FLAIR) (A) and T2-weighted (C) images (arrowheads), which were less evident on T1-weighted (B) and diffusion-weighted (D) images. FLAIR hyperintensities in the cortical regions became shallow and fewer after 3 months (E) (arrowheads). After the second attack that presented with dizziness and left-sided ataxia, the hyperintensities in the pons and left brachium pontis on FLAIR (F), T1-weighted (G), T2-weighted (H), and diffusion-weighted (I) images (arrowheads). The FLAIR hyperintensities in the brainstem became shallow and smaller after 1 month (J).
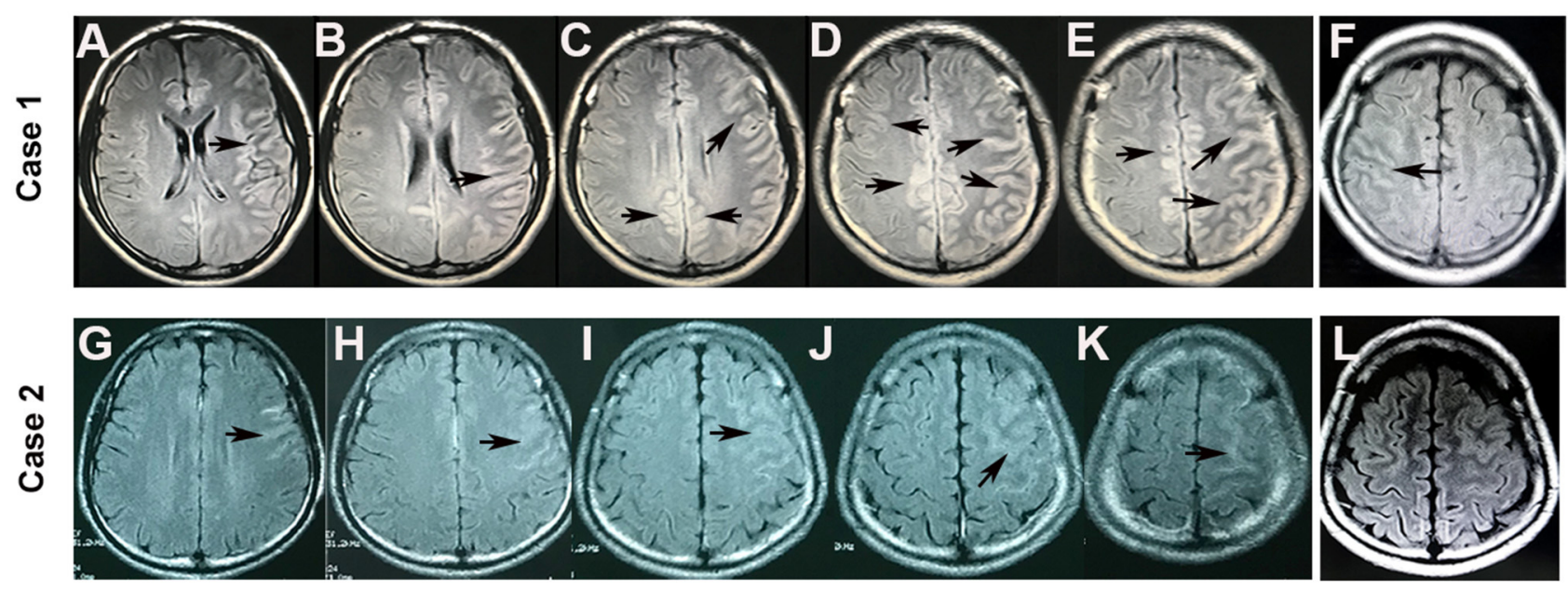

FIGURE 2 | Brain MRI of cortical lesions in cases 1 and 2. FLAIR hyperintensity was seen in the bilateral cerebral cortex in case 1 (A-E) and in the left hemispheric cortical region in case 2 (G-K) (arrowheads). However, the FLAIR hyperintensities in the cortical regions became shallow and fewer after 3 months in case 1 (F) and disappeared after 1 month in case 2 (L).

examination revealed a normal cell count $(3 / \mu \mathrm{l})$ and protein level $(31 \mathrm{mg} / \mathrm{dl})$. The tests for oligoclonal bands and AQP4-IgG in both blood and CSF cultures were negative. MBP in the serum and CSF was not elevated. However, a CBA revealed MOG-IgG in her serum (1:10). She was diagnosed with MOGIgG-associated brainstem encephalitis. After a treatment with intravenous pulse methylprednisolone ( $500 \mathrm{mg} /$ day for 3 days) followed by oral prednisone $(60 \mathrm{mg}$ qd, reduced by $5 \mathrm{mg}$ per week) and mycophenolate mofetil ( $0.5 \mathrm{~g}$ bid), the symptoms gradually improved. The patient was in a stable condition during a 6-month follow-up with $10 \mathrm{mg}$ oral prednisone and $1 \mathrm{~g}$ mycophenolate mofetil daily.

\section{Case 4}

A 22-year-old male patient presented with dizziness and an unsteady gait. He was admitted to a local hospital and received symptomatic treatment for several days. The symptoms persisted and progressively worsened. He felt weakness in 

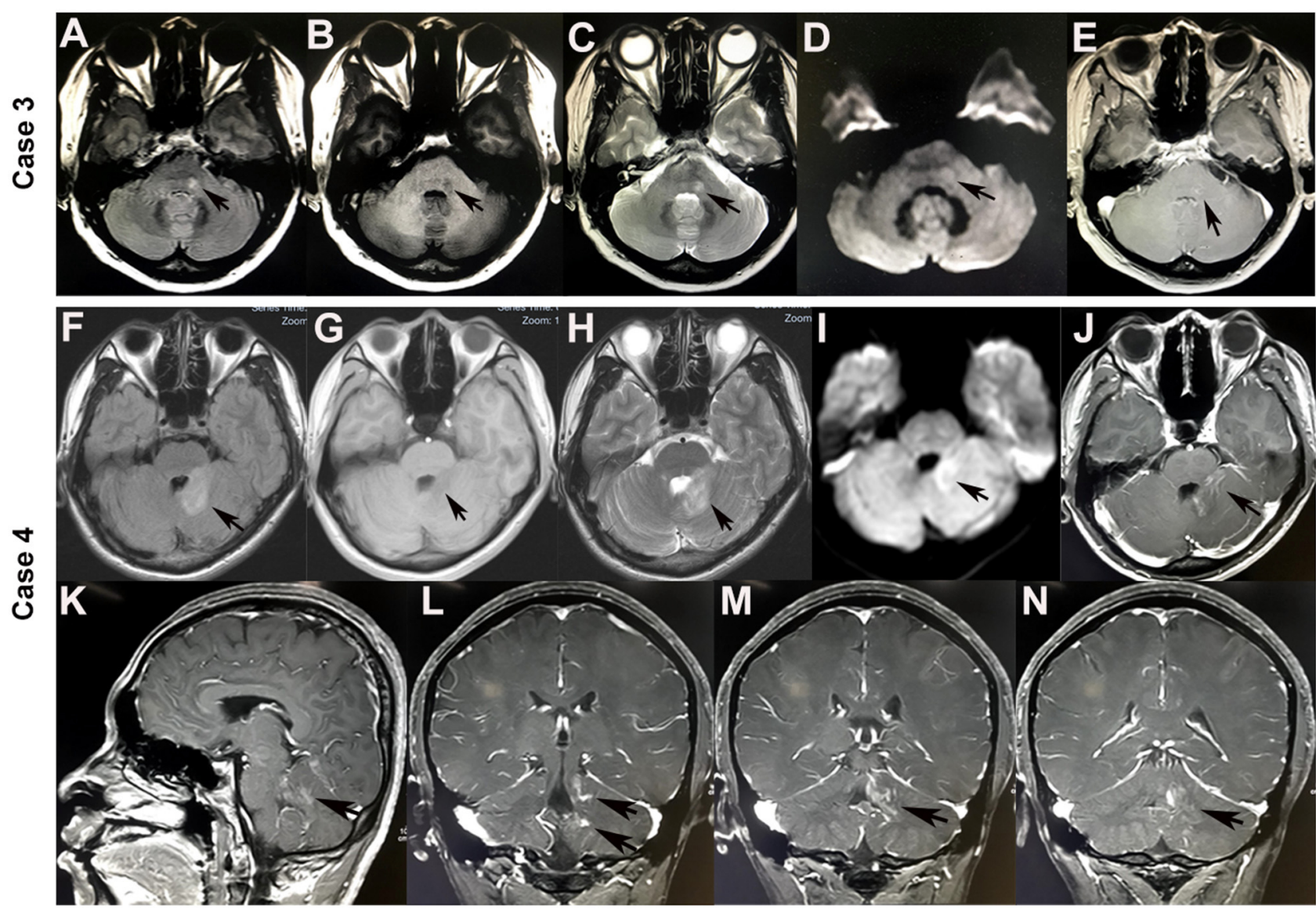

FIGURE 3 | Brain MRI of brainstem lesions in cases 3 and 4. A nodular hyperintense lesion in the left brachium pontis on FLAIR (A), T2-weighted (C), and diffusion-weighted (D) images (arrowheads). However, it was hypointense on the T1-weighted image (B), and with a gadolinium-enhanced edge on the T1-weighted image (E) (arrowheads). Hyperintense lesions in the left brachium pontis, left cerebellar hemisphere, and cerebellar vermis on FLAIR (F), T2-weighted (H), and diffusion-weighted (I) images (arrowheads). However, it was hypointense on the T1-weighted image (G), and with curvilinear post-gadolinium contrast enhancement on the T1-weighted image: axial (J), sagittal (K), and coronal (L-N) (arrowheads).

the left-side limbs and was transferred to our hospital. A nervous system examination discovered mild left-side hemiparesis. Cranial MRI revealed T1-hypointense (Figure 3G), T2-hyperintense (Figure 3H), FLAIR-hyperintense (Figure 3F), and diffusion-hyperintense (Figure 3I) lesions with curvilinear post-gadolinium contrast enhancement (Figures $3 \mathbf{J}-\mathbf{N}$ ) in the left brachium pontis, left cerebellar hemisphere, and cerebellar vermis. The CSF analysis showed a mild increase in leukocytes $(55 / \mu \mathrm{l})$ and normal protein levels. Oligoclonal bands and AQP4-IgG were negative in both blood and CSF. MBP in the serum and CSF was not elevated. However, CBAs detected positive anti-MOG-IgG in CSF (1:1) but negative in serum. Considering the limited reliability of anti-MOG-IgG in CSF and his special image presentation, the patient was diagnosed with probable chronic lymphocytic inflammation with pontine perivascular enhancement responsive to steroids (CLIPPERS) syndrome and received the treatment of high-dose intravenous methylprednisolone (500 $\mathrm{mg} /$ day for 3 days), followed by oral prednisone starting at $60 \mathrm{mg}$ daily with a tapering dose of $5 \mathrm{mg}$ every 1 week. His symptoms of dizziness and hemiplegia greatly improved, and he was discharged after 20 days of treatment. After 5 months, when the prednisone was reduced to $5 \mathrm{mg} /$ day, the patient developed a sudden primary generalized tonic-clonic seizure and was readmitted to our hospital. Neither nervous system examination nor cranial MRI revealed any abnormality. Slow waves were seen in the left prefrontal, frontal, and temporal regions in EEG. Although cell counts $(2 / \mu \mathrm{L})$ and biochemistry of CSF were normal this time, the anti-MOG-IgG was positive in both serum (1:32) and CSF (1:1) as detected by CBAs. He was diagnosed with MOG-IgG-associated encephalitis; therefore, azathioprine (50 mg bid) and antiepileptic drugs (levetiracetam $0.5 \mathrm{~g}$ bid) were added. The patient remained stable during a 6month follow-up with $10 \mathrm{mg}$ oral prednisone, $1.0 \mathrm{~g}$ levetiracetam, and $100 \mathrm{mg}$ azathioprine daily.

\section{DISCUSSION}

Myelin oligodendrocyte glycoprotein (MOG)-IgG-associated disorder (MOGAD) has a specific therapeutic strategy, making early diagnosis and differentiation crucial for developing an 
individualized treatment approach. However, diagnosis at the first demyelinating event remains a challenge due to overlapping and fickle clinical features of MOGAD. Among all clinical manifestations, cerebral and brainstem damage were quite rare disease phenotypes $(<10 \%)$ in both pediatric and adult groups $(7,11,12)$. MOG-IgG-positive unilateral or bilateral cortical encephalitis detected as FLAIR-hyperintense cortical lesions (as observed in cases 1 and 2) was first reported by Ogawa et al. $(5,6,8,13-17)$ in 2017 as an emerging phenotype of MOGAD (Table 1). Brainstem encephalitis is another infrequent presentation except for cortical encephalitis among the clinical spectrum of $\operatorname{MOGAD}(7,13)$. Patients with brainstem involvement account for about 30\% in MOGIgG-associated encephalomyelitis cases, and isolated brainstem encephalitis that occurs without $\mathrm{ON}$ or myelitis is much rarer, accounting for only $1.8 \%$ (18).

Cerebral lesions of MOGAD usually look paler, fewer, and less prominent compared with multiple sclerosis cases and can be observed in both T2-weighted and FLAIR images (8, 19, 20). An important basis for diagnosing MOG-IgG cortical encephalitis is unilateral or bilateral cortical lesions on cranial MRI, which are best depicted on FLAIR images (21). In this study, the lesions in case 1 were slightly hyperintense with a hazy border in the bilateral cortical gray matter on FLAIR (Figures 1A, 2A-E) and T2-weighted images (Figure 1C); the lesions became lighter and fewer after 3 months and were displayed only on the FLAIR image (Figures 1E, 2F). Similar abnormal lesions located in the unilateral cortical gray matter were observed in case 2 only on the FLAIR image at the beginning of the disease (Figures 2G-K) and almost disappeared after a month (Figure 2L). Similar to the cortical lesions observed in cases 1 and 2, FLAIR hyperintensity lesions in the cortex or sulcus are also observed in various pathologic conditions, such as meningitis, leptomeningeal metastasis, acute infarction, subarachnoid hemorrhage, and moyamoya disease (22). Thus, patients with MOG-IgG-positive cortical encephalitis, as in cases 1 and 2 in this study, could have been easily misdiagnosed as other diseases.

Brainstem lesions in MOG-IgG-positive cases usually presented a vague, irregularly bordered focus located in different areas of the brainstem, among which the pons was the most commonly involved and accounted for $84.6 \%$. Other common lesions included medulla oblongata $(57.1 \%)$, cerebellar peduncles (35.7\%), and mesencephalon (14.3\%) successively (18). The clinical presentations varied according to the areas of brainstem involvement, including cranial nerve palsy, hemiplegia, intractable hiccup, respiratory disturbance, balance difficulties, vertigo, and ataxia. In the present study, case 1 developed brainstem encephalitis 3 years after cortical encephalitis with lesions located in the pons and left brachium pontis, leading to dizziness and left-side ataxia. Lesions limited to the left brachium pontis in case 3 resulted in left-side peripheral facial paralysis and decreased touch and pain sensation in the left perioral area. Positive MOG-IgG in serum and steroidresponsiveness in these two patients confirmed the diagnosis of MOG-IgG-associated brainstem encephalitis. In case 4, diffused lesions in the left brachium pontis, left cerebellar hemisphere, and cerebellar vermis led to multiple neurological deficits, including vertigo and hemiplegia. Curvilinear enhancement signal and negative MOG-IgG in serum led to a misdiagnosis of probable CLIPPERS syndrome. However, with a relapse manifesting as a seizure 5 months later and positive MOG-IgG in serum and CSF, he was eventually diagnosed with MOG-IgGassociated encephalitis. The classical MRI features of CLIPPERS syndrome were punctate or curvilinear gadolinium-enhancing lesions, which were the most prominent in the pons (23). In case 4 , the patchy lesions in the left brachium pontis, left cerebellar hemisphere, and cerebellar vermis did not meet the updated CLIPPERS criteria (23). Thus, the presumed diagnosis of CLIPPERS at the first attack was not appropriate. Despite the similar clinical and imaging features of MOG-IgG-related brainstem encephalitis and CLIPPERS (24-26), the diagnostic criteria of CLIPPERS encouraged careful consideration of other possible explanations (23).

Most MOG-IgG are of extrathecal origin; therefore, the presence of MOG-IgG in serum rather than in CSF is recommended as a specific indicator for the diagnosis of MOGAD (4). However, Aoe et al. (27) found that a few patients with MOGAD tested positive for MOG-IgG only in CSF. Patient 4 initially tested positive for MOG-IgG only in CSF, but the detection of MOG-IgG was positive in both serum and CSF when the disease relapsed 5 months later. Thus, we suggest that MOGIgG should be tested in both serum and CSF simultaneously if possible and re-tested when necessary. Valuable data on regular monitoring of antibody titers in patients of MOGAD are scarce. Nevertheless, disease activity and treatment status may contribute to MOG-IgG titers in serum. Higher median titers were observed during the acute phase rather than the remission stage. Meanwhile, immunotherapy may lead to lower titers (28). In cases 1 and 3, we observed a low titer (1:10) of MOG-IgG during the acute episode, the exact reasons for this are not clear, but several speculations can be offered: first, the effect of glucocorticoid immunotherapy. Second, there may be another unknown pathogenic antibody at the same time. Third, as reported in the literature, some patients have relatively low antibody titers during acute attacks (29).

Cerebrospinal fluid (CSF) findings in our cases presented as pleocytosis of predominant lymphocytes and a normal or mildly elevated protein level around the time of an attack, consistent with previous studies on MOG-IgG-associated encephalitis (5, $6,8,13)$. In an international multicenter study, high MBP levels in the CSF were observed in the MOG-IgG-positive cases, suggesting acute myelin damage during attacks (30). However, the CSF MBP levels were non-elevated in our cases, as observed in patients with MOG-IgG-associated cortical encephalitis in several previous case reports $(8,15,31)$. Thus, whether MOG-IgG is directly associated with cortical encephalitis or increased MBP in CSF should be a prerequisite for the diagnosis of MOGAD remains controversial. Notably, positive anti-N-methyl-D-aspartate (NMDA) receptor antibodies were detected in 5/18 of patients in a cohort of MOG-IgG-associated encephalitis (13). This suggested that immune attacks might involve NMDA receptors at the same time. However, antibodies of autoimmune encephalitis in serum and CSF were tested and no 
TABLE 1 | Summary of the case reports about MOG-IgG associated cortical encephalitis.

\begin{tabular}{|c|c|c|c|c|c|c|}
\hline Study & $\begin{array}{l}\text { Number of } \\
\text { patients (Male: } \\
\text { Female) }\end{array}$ & $\begin{array}{c}\text { Mean (median) } \\
\text { age of onset }\end{array}$ & $\begin{array}{l}\text { Main clinical } \\
\text { manifestations }\end{array}$ & $\begin{array}{l}\text { Abnormal } \\
\text { signal in brain } \\
\text { MRI }\end{array}$ & $\begin{array}{l}\text { Other } \\
\text { antibodies }\end{array}$ & Disease course \\
\hline Fujimori et al. (5) & $1 /(1: 0)$ & 46 & $\begin{array}{l}\text { Seizures, diziniss, } \\
\text { paraparesis }\end{array}$ & Bilateral cortex & None & relapsing disease \\
\hline Wang L et al. (13) & $18(10: 8)$ & $21.3(22)$ & $\begin{array}{l}\text { Seizures, ON, } \\
\text { encephalophagy }\end{array}$ & Unilateral cortex & $\begin{array}{l}\text { 5/18 were } \\
\text { NMDAR positive }\end{array}$ & $\begin{array}{l}13 / 18 \text { had a } \\
\text { relapsing disease }\end{array}$ \\
\hline Fujimori J et al. (15) & $6(3: 3)$ & 34 & $\begin{array}{l}\text { Fever, headache, seizures, } \\
\text { paraparesis, lethargy, } \\
\text { memory disturbance }\end{array}$ & $\begin{array}{l}\text { Bilateral cortex } \\
\pm \text { corpus } \\
\text { callosum }\end{array}$ & No data & No data \\
\hline
\end{tabular}

sufficient evidence was found that other associated autoimmune antibodies were responsible for the cortical encephalitis and seizures in cases 1 and 2, as observed in many case series/reports $(5,6,8,14,16,17)$. The cortical damage in these patients was likely triggered by an episode of demyelination caused by MOG-IgG. Another possibility also existed that an unknown auto-antibody might be involved in disease pathogenesis.

At present, although the long-term prognosis of MOGIgG-associated encephalitis is not fully understood, the use of corticosteroids and/or plasma exchange or intravenous immunoglobulin remains the current standard treatment in the acute phase (32). Patients with positive MOG-IgG seem to have milder symptoms of neurological impairment (14) and respond more sensitively to corticosteroids than those with positive AQP4 antibodies (33). However, if appropriate treatments are not given in the early course, the symptoms can gradually deteriorate in some MOG-IgG-positive patients (30). Simultaneously, the tapering of oral prednisone slowly following intravenous methylprednisolone is critical on account of a tendency to relapse for rapid withdrawal $(28,34)$. Longterm immunosuppressive therapy is recommended for patients with recurrence or high risk of recurrence, and mycophenolate mofetil and rituximab are the most commonly used drugs (6). In case 1, the patient did not receive a correct diagnosis and standardized therapy due to limitations in the disease perception and antibody detection methods at the time of the first attack. He also experienced a rapid dose reduction of oral prednisone, leaving intermittent seizures after discharge, and subsequently developed brainstem encephalitis after 3 years. In case 4 , the patient was misdiagnosed for the uncertain significance of MOGIgG in CSF. However, with slow oral prednisone withdrawal, he experienced a relapse representing seizure after 5 months without immunosuppressive agents. Upon experiencing the relapse, cases 1 and 4 were started on mycophenolate mofetil/azathioprine, and they experienced no more events for 28 and 6 months of follow-up, respectively. On the contrary, cases 2 and 3 were diagnosed correctly immediately after the initial attack and recovered completely after the standardized treatment. With the slow oral prednisone and initiation of mycophenolate mofetil, case 2 was stable during 16 months of the follow-up and case 3 remained in clinical remission after 6 months of treatment.

\section{CONCLUSION}

In summary, this study reported four adult patients who suffered from cortical encephalitis with epileptic seizures and/or brainstem encephalitis during the course of the disease, all of whom were MOG-IgG positive. The four cases might enhance the understanding of MOG-IgG-related disorders. Although MOG-IgG-associated cortical/brainstem encephalitis occurs infrequently, it should be taken into consideration in patients who experience sudden seizure or symptoms of brainstem damage and when MRI reveals cerebral cortical or brainstem lesions. Currently, research on MOGAD is limited and requires further exploration.

\section{DATA AVAILABILITY STATEMENT}

The original contributions presented in the study are included in the article/supplementary material, further inquiries can be directed to the corresponding author/s.

\section{ETHICS STATEMENT}

Written informed consent was obtained from the individual(s) for the publication of any potentially identifiable images or data included in this article. 


\section{AUTHOR CONTRIBUTIONS}

All authors listed have made a substantial, direct, and intellectual contribution to the work and approved it for publication.

\section{REFERENCES}

1. Narayan R, Simpson A, Fritsche K, Salama S, Pardo S, Mealy M, et al. MOG antibody disease: A review of MOG antibody seropositive neuromyelitis optica spectrum disorder. Mult Scler Relat Disord. (2018) 25:66-72. doi: 10.1016/j.msard.2018.07.025

2. Peschl P, Bradl M, Hftberger R, Berger T, Reindl M. Myelin oligodendrocyte glycoprotein: deciphering a target in inflammatory demyelinating diseases. Front Immunol. (2017) 8:529. doi: 10.3389/fimmu.2017.00529

3. Ramanathan S, Dale RC, Brilot F. Anti-MOG antibody: the history, clinical phenotype, and pathogenicity of a serum biomarker for demyelination. Autoimmun Rev. (2016) 8:307-24. doi: 10.1016/j.autrev.2015.12.004

4. Jarius S, Paul F, Aktas O, Asgari N, Dale RC, de Seze J, et al. MOG encephalomyelitis: international recommendations on diagnosis and antibody testing. J Neuroinflammation. (2018) 15:1-10. doi: 10.1186/s12974-018-1144-2

5. Fujimori J, Takai Y, Nakashima I, Sato DK, Takahashi T, Kaneko K, et al. Bilateral frontal cortex encephalitis and paraparesis in a patient with anti-MOG antibodies. J Neurol Neurosurg Psychiatry. (2017) 88:5346. doi: 10.1136/jnnp-2016-315094

6. Hamid SHM, Whittam D, Saviour M, Alorainy A, Mutch K, Linaker S, et al. Seizures and encephalitis in myelin oligodendrocyte glycoprotein IgG disease vs aquaporin 4 IgG disease. JAMA Neurol. (2018) 75:6571. doi: 10.1001/jamaneurol.2017.3196

7. Cobo-Calvo A, Ruiz A, Maillart E, Audoin B, Zephir H, Bourre $\mathrm{B}$, et al. Clinical spectrum and prognostic value of CNS MOG autoimmunity in adults: The MOGADOR study. Neurology. (2018) 90:e1858-69. doi: 10.1212/WNL.0000000000005560

8. Ogawa R, Nakashima I, Takahashi T, Kaneko K, Akaishi T, Takai $\mathrm{Y}$, et al. MOG antibody-positive, benign, unilateral, cerebral cortical encephalitis with epilepsy. Neurol Neuroimmunol Neuroinflammat. (2017) 4:e322. doi: 10.1212/NXI.0000000000000322

9. Ikeda T, Yamada K, Ogawa R, Takai Y, Kaneko K, Misu T, et al. The pathological features of MOG antibody-positive cerebral cortical encephalitis as a new spectrum associated with MOG antibodies: a case report. J Neurol Sci. (2018) 392:113-5. doi: 10.1016/j.jns.2018.06.028

10. Tao R, Qin C, Chen $\mathrm{M}, \mathrm{Yu} \mathrm{HH}, \mathrm{Wu} \mathrm{LJ}, \mathrm{Bu} \mathrm{BT}$, et al. Unilateral cerebral cortical encephalitis with epilepsy: a possible special phenotype of MOG antibody-associated disorders. Int J Neurosci. (2020) 130:15. doi: 10.1080/00207454.2020.1720676

11. Jurynczyk M, Messina S, Woodhall MR, Raza N, Everett R, Roca-Fernandez A, et al. Clinical presentation and prognosis in MOG-antibody disease: a UK study. Brain: J Neurol. (2017) 140:3128-38. doi: 10.1093/brain/awx276

12. De Mol CL, Wong Y, van Pelt ED, Wokke B, Siepman T, Neuteboom RF, et al. The clinical spectrum and incidence of anti-MOG-associated acquired demyelinating syndromes in children and adults. Multiple Sclerosis. (2020) 26:806-14. doi: 10.1177/1352458519845112

13. Wang L, Zhang B, Zhou L, Zhang Y, Li H, Li Y, et al. Encephalitis is an important clinical component of myelin oligodendrocyte glycoprotein antibody associated demyelination: a single-center cohort study in Shanghai China. Eur J Neurol. (2019) 26:168-74. doi: 10.1111/ene.13790

14. Nie HB, Gao HF, Li YQ, Shen YY. The clinical features of FLAIRhyperintense lesions in Anti-MOG antibody associated cerebral cortical encephalitis with seizures: case reports and literature review. Medicine. (2021) 100:e26087. doi: 10.1097/MD.0000000000026087

15. Fujimori J, Nakamura M, Yagihashi T, Nakashima I. Clinical and radiological features of adult onset bilateral medial frontal cerebral cortical encephalitis with anti-myelin oligodendrocyte glycoprotein antibody. Front Neurol. (2020) 11:600169. doi: 10.3389/fneur.2020.600169

\section{FUNDING}

This study was supported by the Projects in Science and Technique Plans of Xingtai City (Grant Number 2021ZC152).

16. Kim KH, Cho J, Cho KH, Shin HY1, Kim SW. Anti-myelin oligodendrocyte glycoprotein antibody-positive encephalitis with seizure and unilateral cortical fluid-attenuated inversion recovery-hyperintense lesions. J Clin Neurol. (2021) 17:481-3. doi: 10.3988/jcn.2021.17.3.481

17. Ma GZ, He JZ, Li Y, Xu Y, Hu YX, Cui F. Bilateral meningocortical involvement in anti-myelin oligodendrocyte glycoproteinigg associated disorders: a case report. Front Neurol. (2021) 12:670349. doi: 10.3389/fneur.2021.670349

18. Jarius S, Kleiter I, Ruprecht K, Asgari N, Pitarokoili K, Borisow N, et al. MOG-IgG in NMO and related disorders: a multicenter study of 50 patients. Part 3: Brainstem involvement - frequency, presentation and outcome. J Neuroinflammat. (2016) 13:1-23. doi: 10.1186/s12974-016-0719-Z

19. Akaishi $T$, Konno M, Nakashima I, Aoki M. Intractable hiccup in demyelinating disease with anti-myelin oligodendrocyte glycoprotein (MOG) antibody. Internal Med. (2016) 55:29056. doi: 10.2169/internalmedicine.55.7146

20. Akaishi T, Nakashima I, Sato DK, Takahashi T, Fujihara K. Neuromyelitis optica spectrum disorders. Neuroimaging Clin N Am. (2017) 27:25165. doi: 10.1016/j.nic.2016.12.010

21. Kim S-M, Woodhall MR, Kim J-S, Kim S-J, Park KS, Vincent AF, et al. Antibodies to MOG in adults with inflammatory demyelinating disease of the CNS. Neurol Neuroimmunol Neuroinflamm. (2015) 2:e163. doi: 10.1212/NXI.0000000000000163

22. Maeda M, Yagishita A, Yamamoto T, Sakuma H, Takeda K. Abnormal hyperintensity within the subarachnoid space evaluated by fluid-attenuated inversion-recovery MR imaging: a spectrum of central nervous system diseases. Eur Radiol. (2003) 13:L192-201. doi: 10.1007/s00330-003-1877-9

23. Tobin WO, Guo Y, Krecke KN, Parisi JE, Lucchinetti CF, Pittock SJ. Diagnostic criteria for chronic lymphocytic inflammation with pontine perivascular enhancement responsive to steroids (CLIPPERS). Brain. (2017) 140:241525. doi: 10.1093/brain/awx200

24. Symmonds M, Waters PJ, Küker W, et al. Anti-MOG antibodies with longitudinally extensive transverse myelitis preceded by CLIPPERS. Neurology. (2015) 84:1177-9. doi: 10.1212/WNL.0000000000001370

25. Berzero G, Taieb G, Marignier R, Younan N, Savatovsky J, Leclercq D, et al. CLIPPERS mimickers: relapsing brainstem encephalitis associated with anti-MOG antibodies. Eur J Neurol. (2018) 25:e16-7. doi: 10.1111/ene.13483

26. Obeidat AZ, Block AN, Hooshmand SI. Peppering the pons: CLIPPERS or myelin oligodendrocyte glycoprotein associated disease? Multiple Sclerosis and Relat Disord. (2021) 51:102874. doi: 10.1016/j.msard.2021.102874

27. Aoe S, Kume K, Takata T, Touge T, Kaneko K, Nakashima I, et al. Clinical significance of assaying anti-MOG antibody in cerebrospinal fluid in MOGantibody-associated diseases: a case report. Mult Scler Relat Disord. (2019) 28:165-6. doi: 10.1016/j.msard.2018.12.035

28. Jarius S, Ruprecht K, Kleiter I, Borisow N, Asgari N, Pitarokoili $\mathrm{K}$, et al. MOG-IgG in NMO and related disorders: a multicenter study of 50 patients. Part 2: Epidemiology, clinical presentation, radiological and laboratory features, treatment responses, and longterm outcome. J Neuroinflamm. (2015) 13:280. doi: 10.1186/s12974-0160718-0

29. Jarius S, Ruprecht K, Kleiter I, Borisow N, Asgari N, Pitarokoili $\mathrm{K}$, et al. MOG-IgG in NMO and related disorders: a multicenter study of 50 patients. Part 1: frequency, syndrome specificity, influence of disease activity, long-term course, association with AQP4-IgG, and origin. J Neuroinflammation. (2016) 13:279. doi: 10.1186/s12974-0160717-1

30. Kaneko1K, Sato DK, Nakashimal I, Nishiyamal S, Tanaka S, Marignier R, et al. Myelin injury without astrocytopathy in neuroinflammatory disorders with MOG antibodies. $J$ Neurol, 
Neurosurg Psychiat. (2016) 87:1257-9. doi: 10.1136/jnnp-2015-3 12676

31. Katsuse K, Shimizu G, Saito Sato N, Hatano K, Yagi S, Kimura $\mathrm{T}$, et al. Epilepsia partialis continua as an early sign of antimyelin oligodendrocyte glycoprotein antibody-positive encephalitis. Internal Med. (2020) 59:1445-9. doi: 10.2169/internalmedicine.3 076-19

32. Sara S, Majid K, Santiago P, Izlem I, Michael L. MOG antibodyassociated encephalomyelitis/encephalitis. Multiple Sclerosis. (2019) 25:142733. doi: 10.1177/1352458519837705

33. Kitley J, Woodhall M, Waters P, Leite MI, Devenney E, Craig $\mathrm{J}$, et al. Myelin-oligodendrocyte glycoprotein antibodies in adults with a neuromyelitis optica phenotype. Neurology. (2012) 79:1273-7. doi: 10.1212/WNL.0b013e31826aac4e

34. Chalmoukou K, Alexopoulos H, Akrivou S, Stathopoulos P, Reindl M, Dalakas MC. Anti-MOG antibodies are frequently associated with steroid-sensitive recurrent optic neuritis. Neurol Neuroimmunol Neuroinflammation. (2015) 2:e131. doi: 10.1212/NXI.0000000000000131
Conflict of Interest: The authors declare that the research was conducted in the absence of any commercial or financial relationships that could be construed as a potential conflict of interest.

Publisher's Note: All claims expressed in this article are solely those of the authors and do not necessarily represent those of their affiliated organizations, or those of the publisher, the editors and the reviewers. Any product that may be evaluated in this article, or claim that may be made by its manufacturer, is not guaranteed or endorsed by the publisher.

Copyright (C) 2022 Wang, Yin, Fan, Kang, Wei, Yin and Yin. This is an open-access article distributed under the terms of the Creative Commons Attribution License (CC BY). The use, distribution or reproduction in other forums is permitted, provided the original author(s) and the copyright owner(s) are credited and that the original publication in this journal is cited, in accordance with accepted academic practice. No use, distribution or reproduction is permitted which does not comply with these terms. 